\title{
A co-infecção AI DS/ Hepatite C e a equipe de enfermagem em um hospital especializado
}

\section{Co-infection AI DS/ Hepatitis C and the nursing team in a hospital specialized}

\author{
La co-infección SI DA/ Hepatitis C y el oficio de la equipo de enfermería \\ en un hospital de especialidad
}

Thaís Helena Piai', Rosely Moralez de Figueiredo"

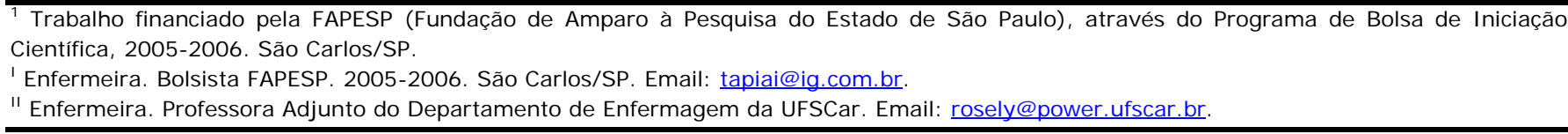

\section{RESUMO}

A Hepatite C é uma doença que afeta cerca de $30 \%$ das pessoas com HIV/aids em todo o mundo. Este trabalho teve por objetivo caracterizar o grau de conhecimento, de percepção, questões correlacionais e de segurança dos profissionais de enfermagem sobre a co-infecção aids/hepatite C, em um hospital especializado em doenças transmissíveis, localizado no interior do Estado de São Paulo - Brasil, no período de outubro de 2005 a novembro de 2006. Trata-se de um estudo exploratório de natureza qualitativa, realizado com 14 profissionais de enfermagem que atuam no referido hospital. Avaliou-se, também, a caracterização dos mesmos quanto à idade, sexo, tempo na profissão e tempo na instituição, por meio de perguntas fechadas. Pôde-se identificar a falta de informação dos profissionais sobre a doença; ausência de política de treinamento e capacitação dos mesmos pela instituição e ainda o desconhecimento das formas de transmissão, gerando a utilização exagerada e sem critério de equipamentos de proteção. É necessária a capacitação e atualização das equipes de enfermagem sobre a doença, em especial os profissionais com mais tempo de formados, contribuindo assim para melhora das condições de trabalho e da qualidade da assistência de enfermagem oferecida.

Descritores: AIDS; Hepatite C; Enfermagem.

\section{ABSTRACT}

Hepatitis C is an illness that affects about $30 \%$ of the people with HIV/aids in the whole world. This work had the objectives of characterize the levels of knowledge, of perception, of related questions, and of security of the nursing professionals about the co-infection aids/hepatitis C, in a southeastern Brazilian hospital specialized in infection disease, during October 2005 a November 2006. This is an exploratory study of qualitative nature, carried out with 14 professionals of nursing who act in the hospital. The professionals were also characterized in relation with their age, sex, time in the profession, and time in the institution, by means of a questionnaire with closed questions. The professionals showed knowledge deficiency in respect of hepatitis $\mathrm{C}$, knowing nothing about transmission forms, and using without criteria, or even not using at all, the protection equipment. The hospital does not offer institutional programmers to educate their employees, although the nursing team knowledge about hepatitis $\mathrm{C}$ is precarious. The urgent necessity of qualification and update politics for the nursing assistance will contribute to the improvement of work conditions and the quality of the nursing assistance offered.

Descriptors: AIDS; Hepatitis C; Nursing.

\section{RESUMEN}

La Hepatite C es una enfermedad que afecta cerca de $30 \%$ de las personas con $\mathrm{VIH} /$ aids en todo el mundo. Este trabajo tuvo por objetivo caracterizar el grado de conocimiento, de percepção, cuestiones correlacionais y de seguridad de los profesionales de enfermería sobre la co-infección aids/hepatite C, en un hospital especializado en enfermedades transmissíveis, en el interior del Estado de São Paulo - Brasil, de octubre 2005 hasta noviembre 2006. Se trata de un estudio exploratório de naturaleza qualitativa, realizado con 14 profesionales de enfermería que tutéan en el referido hospital. Se evaluó, también, la caracterización de los mismos cuánto a la edad, sexo, tiempo en la profesión y tiempo en la institución, por medio de preguntas cerradas. Se pudo identificar la falta de información de los profesionales sobre la enfermedad; ausencia de política de entrenamiento y capacitación de los mismos por la institución y aún el desconhecimento de las formas de transmisión, generando la utilización exagerada y sin criterio de equipamientos de protección. Es necesaria la capacitación y actualización de los equipos de enfermería sobre la enfermedad, en especial los profesionales con más tiempo de formados, contribuyendo así para mejora de las condiciones de trabajo y de la calidad de la asistencia de enfermería ofrecida.

Descriptores: SIDA; Hepatitis C; Oficio de enfermera. 


\section{NTRODUÇÃO}

A Hepatite C é uma inflamação do fígado provocada pelo vírus $\mathrm{HCV}^{(1)}$. Os indivíduos considerados de risco são aqueles que receberam transfusões de sangue e/ou hemoderivados antes de 1992, usuários de drogas intravenosas, pessoas com tatuagens e piercings, alcoólatras, portadores de HIV, transplantados, hemodialisados, hemofílicos, presidiários e sexualmente promíscuos ${ }^{(2)}$.

No Brasil, o Ministério da Saúde, estima que existam 3 milhões de pessoas infectadas pelo vírus HVC. Só na capital paulista são 140 mil infectados ${ }^{(3)}$.

$\mathrm{O}$ fato de ainda não existir vacina contra a hepatite $C$ faz com que ela se torne um dos maiores problemas, atuais, de saúde pública em nosso país. Torna-se também um problema, em especial, para os profissionais da área da saúde (PAS) que se expõem ao risco de infecção diariamente na sua prática profissional $^{(4-5)}$

Dentre os fluidos corporais, tem-se reconhecido - sangue como o mais importante veículo de transmissão do HCV. O risco médio de se adquirir o vírus da hepatite $C$ após exposição percutânea pode variar de 1 a $10 \%$. É importante ressaltar que não existe intervenção específica para prevenir a transmissão do HCV após exposição( ${ }^{(6)}$.

Uma vez co-infectado o paciente sofre com a progressão acentuada da doença hepática. Em geral ocorre uma evolução clínica da doença muito mais agressiva e a manifestação dos efeitos hepatotóxicos da medicação anti-retroviral ${ }^{(1)}$.

Com esta proximidade entre as duas doenças é de se esperar que muitos dos serviços originariamente estabelecidos para atendimento de pacientes com aids, acabem por sua vez, atendendo também a pacientes com hepatite C. Isso acorre sem, necessariamente, ter sido previsto ou planejado pelas instituições.

Conhecer as demandas desta população e estar disposto a ampliar o próprio conhecimento para apoiar, educar, capacitar à equipe e atender melhor os indivíduos co-infectados é uma das metas da enfermeira que atua na área ${ }^{(7)}$.

Conhecer essa demanda implica tanto no desenvolvimento de conhecimento técnico (de como prestar assistência a esse paciente, como evitar exposição ocupacional), como também aspectos relacionais e emocionais dessa interação.

A equipe de enfermagem é extremamente vulnerável à exposição acidental ao vírus da hepatite $C$, uma vez que é o contingente mais numeroso da força de trabalho da saúde, com formação técnica muito heterogênea e ministrando cuidados que envolvem o contato direto com o paciente ${ }^{(8)}$.

A adoção das medidas de biossegurança assume uma importância vital para a redução desses riscos, criando um ambiente seguro, tanto para o profissional, quanto para o usuário dos serviços de saúde. Entretanto, a adoção dessas medidas por parte dos PAS constitui hoje o maior desafio para os profissionais que trabalham com essa temática ${ }^{(8)}$.

O envolvimento emocional estabelecido nessa relação é muito bem descrito na assistência ao paciente com aids. A equipe de enfermagem relata grande desgaste físico e emocional nesse trabalho, associado às internações repetidas, agravamento do estado de saúde, dependência física e morte freqüente do paciente, gerando insatisfação e angústias no profissional ${ }^{(9)}$. Na literatura não há relatos dessa natureza em relação à assistência de enfermagem e a hepatite $C$.

Atualmente, o tratamento da hepatite $\mathrm{C}$ e $\mathrm{HIV/aids} \mathrm{é} \mathrm{realizado} \mathrm{em} \mathrm{nível} \mathrm{ambulatorial,}$ padronizados e custeados pelo Ministério da Saúde ${ }^{(10)}$. Somente são encaminhados aos hospitais especializados os casos mais graves que necessitam de cuidados especiais, devido a questões sociais ou complicações relacionadas às co-infecções. Muitas vezes o motivo da internação é a aids, mas o paciente leva consigo a hepatite $\mathrm{C}$.

Com essa perspectiva em foco e após revisão bibliográfica, onde se identificou pouco conhecimento produzido sobre o tema enfermagem e hepatite $C$ no Brasil( $^{(11)}$, é que se realizou esse trabalho objetivando caracterizar o grau de conhecimento, de percepção, questões correlacionais e de segurança da equipe de enfermagem sobre a co-infecção AIDS/Hepatite C, em um hospital especializado em doenças transmissíveis.

\section{METODOLOGI A}

Trata-se de um estudo exploratório de natureza qualitativa, sendo desenvolvido no período de outubro de 2005 a novembro de 2006. O projeto foi aprovado pelo Comitê de Ética de Pesquisa com Seres Humanos (CEP) da Universidade Federal de São Carlos (Parecer número 108/05) e todos os participantes (sujeitos) da pesquisa assinaram o termo de consentimento livre e esclarecido.

O trabalho foi realizado em um hospital da Secretaria Estadual de Saúde localizado no interior do Estado de São Paulo, para pacientes com doenças transmissíveis. A instituição possui capacidade para 700 leitos, entretanto, várias alas estão desativadas, mantendo sua capacidade de internação em torno de 70 pacientes.

Atualmente a instituição atende pessoas com diagnóstico confirmado de tuberculose e/ou aids, que necessitem de internação prolongada. Porém, a quantidade de pessoas co-infectadas com o vírus da Hepatite C entre a população internada é elevada.

Fizeram parte deste estudo membros da equipe de enfermagem que trabalham na referida instituição, no plantão diurno, que estiveram de acordo e assinaram o termo de Consentimento Livre e Esclarecido. 
Foram realizadas entrevistas individuais, semiestruturadas, com questões abertas sobre temas relevantes ao estudo, que foram gravadas e posteriormente transcritas. A análise foi realizada segundo o modelo de Análise de Conteúdo, categoria Análise Temática(12).

Os resultados foram divididos em duas partes: na primeira foi realizada a caracterização dos profissionais entrevistados, apresentados em seqüência numérica e avaliados segundo: idade, sexo, estado civil, religião, cargo que ocupa, tempo de profissão e tempo de trabalho no hospital (Quadro 1), já na segunda parte os conteúdos foram agrupados por categorias em grandes temas, colocando-se em evidência os assuntos que emergiram durante as entrevistas e ilustrados com trechos das mesmas. Os trechos citados foram transcritos exatamente como foram ditos.

Quadro 1: Caracterização dos profissionais entrevistados segundo idade, sexo, estado civil, religião, cargo, tempo na profissão e tempo na instituição. Américo Brasiliense - SP; 2006.

\begin{tabular}{|c|c|c|c|c|c|c|c|}
\hline $\begin{array}{c}\text { No } \\
\text { Fictício }\end{array}$ & I dade & Sexo & $\begin{array}{c}\text { Estado } \\
\text { Civil }\end{array}$ & Religião & Profissão & $\begin{array}{c}\text { Anos de } \\
\text { profissão }\end{array}$ & $\begin{array}{c}\text { Anos na } \\
\text { I nstituição }\end{array}$ \\
\hline 1 & 42 anos & Feminino & Casada & Católica & Enfermeira & 21 anos & 4 anos \\
\hline 2 & 41 anos & Masculino & Amasiada & Católica & $\begin{array}{c}\text { Aux. } \\
\text { enfermagem }\end{array}$ & 20 anos & 20 anos \\
\hline 3 & 51 anos & Feminino & Casada & Deus & $\begin{array}{c}\text { Aux. } \\
\text { enfermagem }\end{array}$ & 32 anos & 6 anos \\
\hline 4 & 43 anos & Feminino & Casada & $\begin{array}{c}\text { Cristã no } \\
\text { Brasil }\end{array}$ & $\begin{array}{c}\text { Aux. } \\
\text { enfermagem }\end{array}$ & 7 anos & 6 anos \\
\hline 5 & 36 anos & Feminino & Casada & Católica & $\begin{array}{c}\text { Aux. } \\
\text { enfermagem }\end{array}$ & 13 anos & 10 anos \\
\hline 6 & 40 anos & Feminino & Divorciada & Não tem & $\begin{array}{c}\text { Aux. } \\
\text { enfermagem }\end{array}$ & 23 anos & 6 anos \\
\hline 7 & 40 anos & Masculino & Casada & Católica & $\begin{array}{c}\text { Aux. } \\
\text { enfermagem }\end{array}$ & 11 anos & 7 anos \\
\hline 8 & 56 anos & Feminino & Solteira & Católica & $\begin{array}{c}\text { Aux. } \\
\text { enfermagem }\end{array}$ & 30 anos & 7 anos \\
\hline 9 & 44 anos & Feminino & Solteira & $\begin{array}{c}\text { Cristã no } \\
\text { Brasil }\end{array}$ & $\begin{array}{c}\text { Aux. } \\
\text { enfermagem }\end{array}$ & 19 anos & 7 anos \\
\hline 10 & 34 anos & Feminino & Casada & Católica & $\begin{array}{c}\text { Aux. } \\
\text { enfermagem }\end{array}$ & 8 anos & 7 anos \\
\hline 11 & 51 anos & Feminino & Solteira & Católica & $\begin{array}{c}\text { Aux. } \\
\text { enfermagem }\end{array}$ & 15 anos & 6 anos \\
\hline 12 & 47 anos & Masculino & Casado & Católico & $\begin{array}{c}\text { Aux. } \\
\text { enfermagem }\end{array}$ & 25 anos & 7 anos \\
\hline 13 & 43 anos & Feminino & Divorciada & Católica & $\begin{array}{c}\text { Aux. } \\
\text { enfermagem }\end{array}$ & 20 anos & 8 anos \\
\hline 14 & 43 anos & Feminino & Solteira & Católica & Enfermeira & 20 anos & 5 anos \\
\hline
\end{tabular}

Foram destacados 8 temas, sendo (a) falta de conhecimento sobre hepatite $C$, (b) identificação dos trabalhadores com a aids, (c) desinteresse com a coinfecção aids/hepatite $C$, (d) sentimentos enfrentados pelos entrevistados, (e) percepção de risco, (f) assistência de enfermagem propriamente dita, (g) ambiente estressante e (h) Deus como protetor.

\section{RESULTADOS E DISCUSSÃO}

A população estudada é constituída por auxiliares de enfermagem $(78,6 \%)$, sendo $21,4 \%$ masculina, com média de 18,5 anos de experiência profissional e de 7,6 anos de trabalho na Instituição (Quadro 1).

\section{Falta de conhecimento sobre hepatite $\mathbf{C}$}

A falta de conhecimento em relação à hepatite $C$ foi o grande destaque encontrado nas entrevistas, haja vista que muitos dos profissionais não souberam responder as questões ou procuraram desviar-se do assunto, denotando desconhecimento sobre a doença. Ainda as formas de contágio e medidas de proteção da hepatite $C$ foram confundidas com outros tipos de hepatite e, até mesmo com outras doenças como a Tuberculose, que é transmitida por via aérea. Não, o tratamento dela é assim (pausa). O que a gente conhece é pouco né, só sei que é uma área de infecto, onde a gente tem os cuidados que a gente faz... agora conhecimento mesmo já é mais parte dos médicos (risos), que conhece bem ela né, que tem a $C, A, B, C$, né... (E2) 
Tem que se vacinar, se vacinar como a gente faz... eu sou vacinada, minha carteira é atualizada, minha carteira de vacinação... tem vacinação contra hepatite $C$ né? (...) não sei muito do que falar, não sei. (E4)

... a hepatite $\mathrm{C}$ é mais pelo processo respiratório? (E11)

Ações efetivas de educação continuada é a única forma de assegurar a competência da equipe de enfermagem em relação à sua atenção profissional $^{(13)}$. Segundo ainda a autora isto implica em congraçar as necessidades exigidas pelas instituições com as apresentadas pelos enfermeiros, avaliar os meios de promoção e desenvolvimento estratégico e, promover condições materiais e tempo para o cumprimento desta.

Soma-se a isso a escassez de recursos materiais, humanos e didáticos; a desmotivação equipe de enfermagem, o acúmulo de trabalho, e grande demanda no serviço; a falta de integração entre as equipes e o desinteresse da direção de enfermagem e da instituição em fornecer meios para realização de cursos $^{(14)}$.

É fundamental ainda estabelecer um modelo de capacitação alicerçado na aprendizagem significativa e não na simples transmissão de conhecimentos. Deve-se levar em conta as necessidades do serviço de saúde segundo o contexto no qual este está inserido $^{(15)}$.

\section{I dentificação dos trabalhadores com a aids}

A equipe de enfermagem enfrenta em seu cotidiano uma realidade marcante e desgastante ao lidar com o doente co-infectado, pois atribuem a si, a necessidade de proporcionar a este paciente carinho, amor e conselhos, como forma de amenizar o seu sofrimento.

A necessidade de adaptar as formas de atuação, para conseguir acompanhar de perto as inovações tecnocientíficas, gera nos indivíduos um intenso desgaste físico e mental, caracterizando o trabalho como um importante gerador de estresse ${ }^{(16)}$.

A necessidade de treinamento e formação destes trabalhadores é novamente ressaltada, uma vez que os sentimentos envolvidos e os meios de defesa por eles elaborados facilitam a relação entre o profissional e o cliente. Dessa forma pode-se minimizar as angústias e o esgotamento gerado, ademais é importante reforçar a necessidade de um atendimento profissional no âmbito da saúde mental para $\operatorname{ambos}^{(9)}$.

Essa realidade parece comover os entrevistados, de forma que muitos se identificam com o trabalho realizado, dizendo que gostam do que fazem e que é gratificante.

... é gostoso, você pegar um paciente, você vê, por exemplo, o paciente do leito 22 o " $X$ " ele morreu e viveu, então, é gratificante para você... (E3)
Os profissionais inúmeras vezes possuem "comportamento de ajuda" para com os pacientes, dentre estes, ouvir desabafos e dar conselhos, lembrando que não possuem nenhum preparo para tal, o que acaba repercutindo em uma relação puramente pessoal, essa situação permite que ocorra um envolvimento emocional ainda maior entre profissional e paciente, $o$ que se traduz na identificação do profissional com o serviço em que está inserido ${ }^{(9)}$.

Alguns entrevistados identificam-se com seu trabalho através da ajuda ao mais necessitado e sofrido. Para eles as pessoas com problemas socioeconômicos e vícios são as que necessitam de apoio, cuidado e amor.

... é um pessoal bem carente, bem sofrido, a maioria é andarilho, não tem contato familiar... (E5)

... eu gosto assim de dar apoio para eles, de escutar, eles são pessoas que se arrependem muito, que se envolveram com droga, com bebida, muito problema social. (E14)

Os sentimentos apresentados pela equipe de enfermagem são relativamente conflituosos em seu cotidiano. Sentimento de pena em relação à morte, de impotência, agressividade do paciente e obrigação de trabalhar no local, são fatores que provavelmente refletem no profissional ansiedade e angústia ${ }^{(9)}$.

\section{Não valorização da co-infecção aids/ hepatite $\mathbf{C}$}

O despreparo e a não valorização da co-infecção aids/hepatite $C$ são notáveis, uma vez que os profissionais mencionam não se preocupar em saber os resultados dos exames colhidos pelos pacientes, embora saibam que o mesmo é realizado por ser uma rotina do hospital. Dessa forma, a informação ter ou não hepatite $C$ não altera em nada a assistência de enfermagem que o paciente recebe na instituição.

... todos os pacientes que entram aqui fazem todos os exames, inclusive da hepatite $B$, hepatite $C$, e a gente sabe porque vê o exame, mas saber assim por curiosidade eu não tenho não. (E5)

O tratamento da hepatite $C$ ainda não é realizado no hospital, o que é um agravante, pois durante a internação, o paciente co-infectado não recebe medicações específicas para o vírus $C$.

\section{Sentimentos enfrentados pelos entrevistados}

o envolvimento emocional estabelecido nessa relação é muito bem descrito na assistência ao paciente com aids. Profissionais de enfermagem relatam grande desgaste físico e emocional nesse trabalho, associado às internações repetidas, agravamento do estado de saúde, dependência física e morte freqüente do paciente gerando insatisfação e angústias no profissional ${ }^{(9)}$. Na literatura não há relatos dessa natureza em relação à assistência de enfermagem e a hepatite $\mathrm{C}$. 
Medo

A enfermagem por ser o maior contingente de trabalhadores exercendo cuidados que envolvem o contato direto com o paciente, permite que esse profissional exponha-se ao risco de contrair inúmeras doenças, dentre estas, algumas que ainda não têm cura como a aids e a hepatite $C$. Como conseqüência deste fato vive diariamente sob pressão e medo com os riscos oferecidos pelo trabalho.

... a gente detalha mais os movimentos, né! Você fica mais atenta parece, mas o certo seria você estar atenta aqui e atenta lá fora, não tem... parece que aqui você programa mais teus movimentos... (E1)

O nível de pressão exercido pela organização do trabalho, a exigência de maior produtividade, associada à redução contínua do contingente de trabalhadores, à pressão do tempo, o aumento da complexidade das tarefas, além de expectativas irrealizáveis e relações de trabalho tensas e precárias, podem gerar tensão, fadiga e esgotamento profissional. Esses fatores psicossociais podem ser responsáveis por situações de estresse relacionado com o trabalho ${ }^{(16)}$.

Quando eu entro do portão para dentro, então eu já sei o local onde eu estou entrando, e sei em quem que eu vou mexer. Então, a gente tem um... já tem que estar mais atento, você não vai descuidar, para que você não seja também um deles. (E7)

É certo que as situações que oferecem medo e estresse propiciam o erro, permitindo que acidentes com pérfurocortantes aconteçam. Sendo assim, a angústia gerada nestes profissionais é ainda maior, pois já sabem que os pacientes que estão cuidando são co-infectados.

\section{Medo de agressão}

Os pacientes encontrados em hospitais especializados em doenças infecciosas, como este, têm perfis muito semelhantes, uma vez que grande parte são dependentes químicos, pessoas com problemas com a justiça e apresentam mudanças no comportamento, isso leva aos profissionais certa insegurança, medo de serem agredidos ou de acabarem se perfurando.

Eu tenho mais medo de me perfurar, é o que eu mais me preocupo, assim, que eu tenho medo, a gente sempre toma cuidado, mas a gente nunca sabe né. Porque aqui é um setor... eles são todos imprevisíveis, eles estão bem, de repente não estão. (E10)

Esse perfil populacional costuma gerar reações de medo e resistência nos profissionais de saúde uma vez que ocorre um confronto de valores individuais, culturais, religiosos e éticos ${ }^{(9)}$.

\section{Medo e a família}

O trabalho da enfermagem é marcante por caracterizar-se como a profissão que em sua maioria é ocupada por pessoas do sexo feminino e, pela especificidade das ações que desenvolvem no seu dia-a-dia. As profissionais desta área convivem com a dinâmica das suas atividades diárias e, ao mesmo tempo, gerenciam suas vidas como pessoas, esposas e mães ${ }^{(17)}$.

Pensava na minha família... de passar alguma coisa assim para eles, para os meus filhos (...) eu medo mesmo, de eu morrer, tal... não, nunca tive, eu só pensava assim... ter medo de passar para os meus filhos, ou eles saberem e terem algum preconceito comigo, entendeu? Só que eu pensei só nessa parte viu, em mim não... (E3)

O medo do contágio é evidente entre a equipe de enfermagem que lida diretamente com os doentes co-infectados. Tanto por trabalharem sob um estresse contínuo, ainda são pressionados com o risco de levarem doenças para suas famílias. Essa situação pode causar pânico entre os profissionais gerando dificuldades de contratação de pessoal e até mesmo abandono de emprego por pressão de familiares $^{(9)}$.

\section{Percepção de Risco}

A equipe de enfermagem é extremamente vulnerável à exposição acidental ao vírus da hepatite $C$, uma vez que fazem parte de uma classe bastante numerosa, heterogênea e que prestam assistência que envolve cuidados diretos com o paciente. Sendo assim, é necessário que estes adotem medidas de biossegurança para que haja redução desses riscos.

O uso de equipamentos de proteção individual é bem aceito pelos profissionais entrevistados, uma vez que estes são usados para qualquer cuidado, mesmo que não envolva contato direto com o paciente como levar um copo d'água ou entrar no quarto para conversar.

... a partir do momento que eu vou entrar no quarto, eu vou paramentada vou sempre de avental, de máscara e de luva, independente se vou pegar um copo de água para ele, se ele chama... se eu passo e eles chamam eu já vou de máscara e de luva, nunca vou sem luva e sem máscara no quarto, nunca entrei...(E9)

A todo e qualquer paciente internado em hospital deveriam ser adotadas as medidas de precauções padrão e somente, quando necessário, incluídas as precauções baseadas na transmissão(18). Aqui tem os paramentos: é o avental descartável, a gente tem luva, a gente tem as máscaras, tem as luvas de procedimento. Para qualquer paciente vocês usam a máscara N95? Para qualquer paciente.... (E7)

O uso indiscriminado de equipamentos de proteção, contrário do que parece, não aumenta a segurança do profissional. A falsa proteção gerada no seu uso sem critérios acaba banalizando os riscos, além de elevar o custo para a instituição. Outro aspecto observado no hospital é o uso indiscriminado 
da máscara N95, indicada especificamente para precauções aéreas, mas utilizadas na instituição por todos os profissionais e em qualquer situação.

\section{Assistência de Enfermagem propriamente dita}

A assistência de enfermagem nem sempre é o enfoque do profissional que cuida dos co-infectados do hospital, pois para muitos destes o significado para os "cuidados" são as medidas de proteção usadas para minimizar os riscos, poucos se referem aos "cuidados" como assistência de enfermagem propriamente dita. A preocupação ainda está centrada apenas na sua própria proteção e não conseguem enxergar as necessidades específicas do paciente trazidas pela doença.

É, você tem que tomar os cuidados, né, então, por exemplo, o hospital que dá o material, aí o que resta para você é tomar o cuidado, né, não se perfurar com agulha ou qualquer outro objeto que usou nele. Como cuidado com a secreção, essas coisas. (E12)

O risco de aquisição ocupacional do vírus da hepatite $C$ por trabalhadores da área da saúde, é um fato concreto $^{(5)}$, isto reforça essa preocupação como prioridade para este profissionais. Atitudes de treinamento e disponibilização de equipamentos de proteção e abordagem desse tema em ações educativas podem contribuir para a ampliação das preocupações desse profissional onde a assistência de enfermagem prestada ao paciente também fosse considerada prioridade.

\section{Ambiente estressante}

Os profissionais referem ter afinidade com o trabalho no hospital, haja vista que muitos relatam que gostam do que fazem e sentem-se satisfeitos em cuidar dos pacientes, porém em muitas falas destes, pode-se observar certo receio em falar do hospital, relatando alguns descontentamentos enfrentados.

Dentre os maiores problemas relatados pelos profissionais podem-se destacar a falta de profissionais de enfermagem e outros, estrutura física do prédio em condições precárias e ambientes de trabalho estressante.

... quando eu entrei aqui tantos funcionários que se trombava um com o outro, então, os cargos hoje tão todos desviados, eu quero desviar o meu também e não consigo. Então, existe muita falta de funcionário e os poucos que ficam tá todo mundo estressado... (E3)

É um trabalho difícil, é pesado, você sai daqui sugada, mas eu gosto do que eu faço.(E14)

A enfermagem hospitalar é caracterizada por um trabalho contínuo, com atividades em 24 horas, sem descanso, com extensa carga horária semanal, realizado cotidianamente, incluindo os dias de feriado e festas comemorativas. Este trabalho mesmo sendo necessário, possui pouco reconhecimento social, é desvalorizado perante os demais profissionais da saúde, inclusive pelos pacientes ${ }^{(17)}$.

\section{Deus como protetor}

A figura de Deus é usada pelos profissionais de enfermagem da instituição como alguém que os protege dos riscos encontrados na prática profissional. Deus seria o "escudo" e a confiança para continuarem no trabalho sem que nada de mau os atingissem.

Quando questionados sobre os riscos, e se já tinham sofrido algum acidente ocupacional com materiais perfurantes ou cortantes, muitos buscaram minimizar os efeitos deste risco dizendo que são protegidos por Deus.

... eu acho que eu sou protegido por Deus. (E7)

A religiosidade sempre esteve ao lado da história da humanidade, quer seja tentando explicar o desconhecido ou dando sentido às inquietações humanas, tornando-se, entretanto muito mais presente nos momentos de sofrimento ${ }^{(9)}$.

\section{CONSI DERAÇÕES FI NAIS}

A falta de informação da equipe de enfermagem sobre a doença é um fato marcante encontrado nas entrevistas.

Os hospitais que atendem pacientes com diagnósticos de aids acabam acolhendo também os co-infectados com hepatite C. Isto ocorre sem possuírem recursos ou preparo especial para tal. Os co-infectados não têm atenção específica para hepatite $C$, na instituição estudada.

O medo da doença e sua transmissibilidade é a preocupação mais intensa da equipe de enfermagem, haja vista que muitos ainda não conseguem avaliar a assistência de enfermagem propriamente dita. Isso se traduz no fornecimento de cuidados de enfermagem estritamente necessários, culminando no isolamento desnecessário e utilização exagerada de equipamentos de proteção como máscaras, luvas duplas, aventais. Além disso, estes trabalhadores também se preocupam com os familiares e as doenças que podem levar para casa.

Para ampliar os conhecimentos da equipe de enfermagem é importante que haja formação e treinamento das mesmas, isto permite maior segurança para realização dos cuidados, bem como melhoria na atenção ao paciente co-infectado. Sendo este momento propício para compartilharem suas dúvidas, angústias e receios.

Outro aspecto que deve ser lembrado é a necessidade de maior interação entre os diferentes serviços de saúde que atendem HIV/aids, uma vez que os mesmos atenderão compulsoriamente os coinfectados.

A hepatite $C$ é um dos grandes problemas de saúde pública da atualidade. Trabalhos que avaliem suas diferentes facetas devem ser estimulados. 


\section{REFERÊNCI AS}

1. Strauss E. Hepatite C. Rev. Soc. Bras. Med. Trop. $2001 ; 34(1): 69-82$.

2. Ferreira CT, Silveira TR da. Hepatites virais: aspectos da epidemiologia e da prevenção. Rev. bras. epidemiol. 2004; 7(4): 473-487.

3. Centro de Vigilância Epidemiológica - CVE/SES-SP [Internet]. São Paulo: Secretaria de Estado da Saúde (BR) [update 2009 mar 03, cited 2009 mar 05]. Centro de Vigilância Epidemiológica "Prof. Alexandre Vranjac". Available from: http://www.cve.saude.sp.gov.br/.

4. Malaguti SE, Hayashida M, Canini SRMS, Gir E. Enfermeiros com cargos de chefia e medidas preventivas à exposição ocupacional: facilidades e barreiras. Rev. esc. enferm. USP. 2008;42(3):496503.

5. Gir E, Caffer-Netto J, Malaguti SE, Canini SRMS, Hayashida M, Machado AA. Acidente com material biológico e vacinação contra hepatite $B$ entre graduandos da área da saúde. Rev Latino-am Enfermagem. 2008; 16(3): 401-406.

6. Resende MR, Fortaleza CMCB. Risco ocupacional entre profissionais da área de saúde e medidas de proteção. In: Colombrini MRC, Figueiredo RM, Paiva MC de, editores. Leito-dia em aids: uma experiência multiprofissional. São Paulo: Editora Atheneu; 2001.

7. Brennan C. Treatment of hepatitis $C$ virus in the coinfected patient. Journal of the Association of Nurses in AIDS Care. 2003; 14(5Suppl):52S-79S.

8. Nichiata LYI, Gir E, Takahashi RF, Ciosak SI. Evolução dos isolamentos em doenças transmissíveis: os saberes na prática contemporânea. Rev. esc. enferm. USP. 2004;38(1):61-70.

9. Figueiredo RM, Turato ER. Needs for assistance and emotional aspects of caregiving reported by AIDS patient caregivres in a day-care unit in Brazil. Issues in Mental Health Nursing. 2001;22:633-643.

10. Secretaria de Estado da Saúde (SP). Coordenação dos Institutos de Pesquisa. Centro de Vigilância Epidemiológica "Prof. Alexandre Vranjac". Guia de Orientações Técnicas Hepatite B e C. São Paulo (Brasil): Secretaria de Estado da Saúde (SP); 2002.

11. Figueiredo RM, Piai TH. Hepatite C e enfermagem: revisão de literatura. REME - Rev. Min. Enferm. 2007;11(1): 86-89.

12. Bardin L. Análise de Conteúdo. Lisboa: Edições 70; 1977.

13. Koizumi MS, Kimura M, Miyadahira AMK, Cruz D de ALM da, Padilha KG, Sousa RMC de, et al. Educação continuada da equipe de enfermagem nas UTIs do município de São Paulo. Rev Latino-am Enfermagem. 1998; 6(3): 33-41.

14. Silva JA, Ogata MN, Machado MLT. Capacitação dos trabalhadores de saúde na atenção básica: impactos e perspectivas. Rev. Eletr. Enf. [Internet]. 2007 [cited 2009 feb 16];9(2):389-401. Available from:

http://www.fen.ufg. br/revista/v9/n2/pdf/v9n2a08.pdf. 15. Miquelim JDL, Carvalho CBO, Gir E, Pelá NTR. Estresse nos profissionais de enfermagem que atuam em uma unidade de pacientes portadores de hiv-aids. DST - J bras Doenças Sex Transm. 2004;16(3):2431.

16. Davim RMB, Torres GV, Santos SR dos. Educação continuada em enfermagem: conhecimentos, atividades e barreiras encontradas em uma maternidade escola. Rev Latino-am Enfermagem. 1999; 7(5): 43-9.

17. Spindola T, Santos RS. Mulher e trabalho - a história de vida de mães trabalhadoras de enfermagem. Rev Latino-am Enfermagem. 2003; 11(5): 593-600.

18. Figueiredo RM, Leite $C$. As práticas de precauções/isolamento a partir do diagnóstico de internação em unidade de moléstias infecciosas. Rev. Eletr. Enf. [Internet]. 2006 [cited 2009 feb 16];8(3):358-62. Available from: http://www.fen.ufg.br/revista/revista8_3/v8n3a06.htm.

Artigo recebido em 30.10.07.

Aprovado para publicação em 31.03.09. 